\title{
Cognition- and Anxiety-Related Behavior, Synaptophysin and MAP2 Immunoreactivity in the Adult Rat Treated with a Single Course of Antenatal Betamethasone
}

\author{
MATTEO BRUSCHETTINI, DANIËL L.A. VAN DEN HOVE, SILVIE TIMMERS, MAAIKE WELLING, HELLEN P. STEINBUSCH,
} JOS PRICKAERTS, DIEGO GAZZOLO, CARLOS E. BLANCO, AND HARRY W.M. STEINBUSCH

\begin{abstract}
Department of Psychiatry and Neuropsychology [M.B., D.L.A.H., S.T., M.W., H.P.S., J.P., H.W.M.S.], Division of Neuroscience, Department of Pediatrics [M.B., D.L.A.H., H.P.S., C.E.B.], Research Institute Growth and Development (GROW), European Graduate School of Neuroscience (EURON) [M.B., D.L.A.H., J.P., H.W.M.S.], University of Maastricht, 6200 MD Maastricht, The Netherlands;

G. Gaslini Children's Hospital [M.B., D.G.], University of Genoa, 16167 Genoa, Italy; Maternal Fetal and Neonatal Health [D.G.], G. Garibaldi Hospital, 95100 Catania, Italy; Laboratory of Neuroscience [M.B., D.G.], IRCCS-CN, Bonino-Pulejo, 98124, Messina, Italy
\end{abstract}

\begin{abstract}
We investigated the effects of a single course of antenatal betamethasone on cognition- and anxiety-related behavior and synaptophysin and microtubule-associated protein 2 (MAP2) immunoreactivity in the adult rat hippocampus. On d 20 of gestation, pregnant rats were injected with either 1) $170 \mu \mathrm{g} / \mathrm{kg}$ body weight of betamethasone ("clinically equivalent dose," equivalent to $12 \mathrm{mg}$ twice, $24 \mathrm{~h}$ apart); 2) half this dose; or 3) vehicle. Cognition- and anxiety-related behavior of the offspring was analyzed at an age of 5 mo using the Morris water maze, object recognition task, and open field test. Subsequently, synaptophysin and MAP2 immunoreactivity were measured in the hippocampus. We report no detrimental effects of antenatal betamethasone on cognition- and anxiety-related behavior and synaptophysin immunoreactivity in the adult rat. On the other hand, MAP2 immunoreactivity was decreased by betamethasone in males, suggesting a permanent impairment in the hippocampus. Interestingly, the lower dose appears to have less influence in terms of growth restriction, known to be associated with an increased risk of disease in adulthood. Further research might elucidate whether the betamethasone effect on hippocampal neurons persists later in life and could affect the aging process increasing the risk for neuropathology of the adult. (Pediatr Res 60: 50-54, 2006)
\end{abstract}

A ntenatal glucocorticoids are widely used to prevent respiratory distress syndrome in case of threat of preterm delivery (1). Although beneficial for lung maturation, this treatment causes a wide range of side effects in humans, including a reduction in weight and head circumference at birth (2), and in impairment in both circulating glucocorticoid bioactivity (3) and cortisol response to stressors (4). Animal studies showed that antenatal glucocorticoids affect the hypothalamic-pituitary-adrenal (HPA) axis (5), an impairment of which has been linked to the development of anxiety and mood disorders (6). Further, this treatment has been reported to reduce DNA content (7), cell proliferation rate (8), synaptic

Received December 2, 2005; accepted March 12, 2006

Correspondence: Matteo Bruschettini, M.D., Department of Psychiatry and Neuropsychology, Division of Neuroscience, Faculty of Medicine, Maastricht University, P.O. Box 616, 6200 MD Maastricht, The Netherlands; e-mail: m.bruschettini@np.unimaas.nl

DLAH and JP are supported by the EU Framework 6 Integrated Project NEWMOOD (LSHM-CT-2004-503474).

DOI: $10.1203 / 01 . p d r .0000220349 .41675 .92$ density (9), and neurotrophic factor concentrations within the neonatal brain (10) and induces neural degeneration within the developing hippocampus (11). However, little is known about the consequences for later life, in particular, the effects on cognition- and anxiety-related behavior are unclear $(5,12)$.

The presynaptic marker protein synaptophysin is a calciumbinding protein that plays an important role in the generation and maintenance of small vesicle membranes and their interaction with cytoskeletal elements. Decrease in the synaptophysin IR is associated with brain injury and aging, possibly reflecting functional disturbances of synaptic transmission (13). The neuronal MAP2 determines stability and arrangement of neuronal microtubules. Loss of MAP2 IR after brain injury correlates with neuronal degeneration (14) and is associated with age-related impairment of synaptic plasticity, cognition, and memory functions. Interestingly, both synaptophysin IR and MAP2 IR have been reported to be decreased in the hippocampus shortly after betamethasone injection $(9,15,16)$, whereas the long-term consequences on these parameters are not known. As the hippocampus is involved in learning and memory processes, we aimed to evaluate the effects of a single course of antenatal betamethasone as used in clinical practice on 1) cognition- and anxiety-related behavior and 2) synaptophysin IR and MAP2 IR in the adult rat hippocampus.

\section{METHODS}

Animals. The animal study was approved by the Animal Ethics Board of the University of Maastricht, The Netherlands.

Pregnant Fisher 344 dams (Charles River, Maastricht, The Netherlands; pregnancy confirmed by vaginal plug) were delivered to our animal facility on d 14 of gestation (embryonic d 14, E14). The animals were kept under standard laboratory conditions with $12 \mathrm{~h}$ light/12 h dark and standard rat chow and water ad libitum.

Abbreviations: CD, clinically equivalent dose (corresponding to $12 \mathrm{mg}$ in the woman); DG, dentate gyrus; HD, half CD (corresponding to $6 \mathrm{mg}$ in the woman); IR, immunoreactivity; MAP2, microtubule-associated protein 2; MWM, Morris water maze; OF, open field test; ORT, object recognition task 
Dams were injected subcutaneously twice $8 \mathrm{~h}$ apart with either 170 or 85 $\mu \mathrm{g} \mathrm{kg}^{-1}$ betamethasone, representing a clinically equivalent dose $(\mathrm{CD}$, corresponding to a single course of $12 \mathrm{mg}$ betamethasone twice, $24 \mathrm{~h}$ apart in the woman) and half of this dose (HD), respectively, as described in a previous study (10). Betamethasone injection occurred at E20 when brain development in the rat is comparable to very preterm human infants (17).

All the dams delivered on E22, corresponding to postnatal d 0 (P0). Pups were labeled with paw cut and cross-fostered to dams that had given birth on the same day and had received vehicle-only treatment (to prevent a possible betamethasone effect on maternal behavior). All the litters were culled at four males and four females per dam, and the cages cleaned once a week. At P21, the pups were weaned and housed together (two rats of the same gender and experimental group per cage) up to 5 mo of age.

Behavioral tests. At an age of $5 \mathrm{mo}$, rat performance was assessed in the MWM (spatial memory), ORT (object memory), and OF (anxiety).

Morris water maze. This test was conducted in a tank (diameter, $1.53 \mathrm{~m}$ ) in which an escape platform (diameter, $11 \mathrm{~cm}$ ) was submerged $(1.5 \mathrm{~cm}$ ) below the surface of the water. The rats started facing the wall of the tank from one of out four different, randomly chosen, starting positions, and were trained to find the invisible platform at a fixed invisible position in the water tank. Abundant spatial cues were provided by the furniture in the room and the presence of the experimenter. A videocamera installed above the pool and attached to a PC that registered automatically (EthoVision Color Pro, Noldus, Wagenengen, The Netherlands) the movements of a rat. A trial lasted until a rat had found the platform or until $60 \mathrm{~s}$ had elapsed. If a rat did not find the platform within $60 \mathrm{~s}$, it was placed on the platform for $3 \mathrm{~s}$ and then removed from the water tank. The rats were given two trials a day for $4 \mathrm{~d}$ and the time between subsequent trials was $10 \mathrm{~min}$. Escape latency was averaged per rat per session of two trials. A probe trial lasting $60 \mathrm{~s}$, during which the platform was removed from the water tank, was given after the last trial to reveal whether the animals had learned the position of the platform. In the probe trial, all rats started facing the wall of the tank from the position opposite to the position of the removed platform.

Objects recognition task. The rats were placed in the arena (diameter, 83 $\mathrm{cm}$; height, $40 \mathrm{~cm}$ ) facing the transparent segment of the wall, made of polyvinyl chloride. A testing session comprised two trials of 3 min each. During the first trial (T1), the apparatus contained two identical objects. After the first exploration period, the rat was put back in its home cage for $1 \mathrm{~h}$ and then was put back in the arena for the second trial (T2), but now with two dissimilar objects, a familiar one (the sample) and a new one. The duration of exploring each object in $\mathrm{T} 1$ and $\mathrm{T} 2$ was recorded manually with a personal computer. Exploration was defined as follows: directing the nose to the object at a distance of no more than $2 \mathrm{~cm}$ and/or touching the object with the nose. Sitting on the object was not considered as exploratory behavior. To avoid the presence of olfactory trails, the objects were always thoroughly cleaned. Moreover, each object was available in triplicate so that none of the two objects from the first trial had to be used as the familiar object in the second trial. In addition, all combinations and locations of objects were used in a balanced manner to reduce potential biases due to preferences for particular locations or objects. The light intensity $(201 \mathrm{x})$ was equal in the different parts of the apparatus. We used four different sets of objects that could not be displaced by a rat. Testing sessions were given on three consecutive days. The basic measures were the total exploration time of both objects during T1 and T2, E1 and E2, respectively. A discrimination index, D2, was calculated as following: $\mathrm{D} 2=($ exploration new object - exploration familiar object $) /$ total exploration time. In addition, each experimental group was compared with the level expected by chance, i.e., equal amount of time spent on the new and the old object $(\mathrm{D} 2=0)$. In the last day of test trial, a longer interval $(4 \mathrm{~h})$ between $\mathrm{T} 1$ and $\mathrm{T} 2$ was used to discriminate cognition differences between experimental groups.

Open field. This test was conducted in a square, clear Plexiglas box (100 $\times 100 \times 30 \mathrm{~cm}$ ) with an open top and a dark floor, in a dimly illuminated room. The arena was subdivided into a central zone $(64 \times 64 \mathrm{~cm})$, corners $(16 \times 16 \mathrm{~cm})$ and walls $(16 \times 64 \mathrm{~cm})$. As a rat was placed in the centre of the $\mathrm{OF}$, the position of the animals were registered automatically via a PC (EthoVision Color Pro, Noldus) through a videocamera installed $250 \mathrm{~cm}$ above the field for $5 \mathrm{~min}$. Testing was carried out for $4 \mathrm{~d}$, consisting of a single 5-min session per day.

MAP2 IR and Synaptophysin IR. At an age of $6 \mathrm{mo}$, rats were anesthetized by a sodium pentobarbital injection $(60 \mathrm{mg} / \mathrm{kg}$ i.p.). Subsequently, the brains of the rat were fixated by means of a transcardial perfusion with a flush of tyrode solution $\left(\mathrm{pH} 7.4,4^{\circ} \mathrm{C}\right)$ followed by a Somogyi fixation. After dissection of the brain, it was postfixed in the same fixating for $2 \mathrm{~h}$. Thereafter, the brain segments were cryoprotected in $20 \%$ sucrose in Trisbuffered saline (TBS, $\mathrm{pH}$ 7.4) for $48 \mathrm{~h}$ and then were frozen by means of $\mathrm{CO}_{2}$ and stored at $-75^{\circ} \mathrm{C}$ for further analysis. Sections of $30 \mu \mathrm{m}$ were cut with the cryostat at levels of the hippocampus (between $1.60 \mathrm{~mm}$ and $-5.80 \mathrm{~mm}$ from bregma) for synaptophysin and MAP2 staining. The serial brain slices of 30 $\mu \mathrm{m}$ were stained with MAb against synaptophysin (mouse anti-synaptophysin, 1:2000, Chemicon International, Temecula, CA) and MAP2 [monoclonal anti-MAP2 $(2 \mathrm{a}+2 \mathrm{~b})$, Clone AP -20, 1:1000; Sigma Chemical Co., St. Louis, $\mathrm{MO}$ ] by means of the $\mathrm{ABC}$ technique. Before all antibody incubations, washing steps were carried out using subsequently TBS-T (10 min), TBS (10 $\mathrm{min})$, and TBS-T $(10 \mathrm{~min})$. Next, the tissue slides were incubated with $5 \%$ normal donkey serum (NDS), to minimize the background reactivity. After administration of the primary antibody as mentioned above, the brain slices were kept overnight at $4^{\circ} \mathrm{C}$. Before the secondary biotinylated antibodies were added for a 1-h incubation, the slices were repeatedly washed with TBS-T and TBS. Subsequently, the brain slices were incubated with a preformed avidinhorseradish peroxidase complex for $1 \mathrm{~h}$. Immunostaining was developed by adding DAB solution to the tissue slides, which resulted in a brown precipitate. To optimize the color development, the brain slices were washed with Tris- $\mathrm{HCl}$ before the administration of the DAB solution. The staining procedure was finished by washing the slices three times with TBS $(10 \mathrm{~min})$ and placing the brain slices on glass slides, which were allowed to dry for $24 \mathrm{~h}$. Thereafter, the slides were dehydrated in a $70 \%$ ethanol line followed by enclosure with Pertex.

Quantification of the tissue staining was performed morphometrically by the same investigator who was blinded to the experimental protocol. IR of synaptophysin and MAP2 were estimated within different layers of the hippocampus. The synaptophysin staining was examined in the hippocampal subfields CA1 and CA3 of the stratum radiatum and the dentate gyrus (DG), whereas MAP2 IR was examined in the stratum radiatum (R) and the stratum moleculare (M). For MAP2 IR, slices between -3.14 and $-5.80 \mathrm{~mm}$ from the bregma were examined and averaged.

For each animal and histochemical staining, multiple areas were measured and the results were averaged. Namely, for the synaptophysin staining, three areas were measured in the CA1 subfield, whereas for the CA3 and the DG two were measured. On the other hand, the MAP2 IR measurements were made in one area, both for the stratum radiatum and the stratum moleculare. The number of animals in each treatment group ranged from six to eight for both the synaptophysin and the MAP2 staining. An Olympus Ax70 microscope equipped with a cooled CCD Olympus Digital video camera F-view was used to detect IR by an image analysis system, slightly modified for detection of grayscale punctae (AnalySIS, Soft Imaging System, Münster, Germany). All measurements were performed on a single focal plane. Shading error correction was performed before measurements to correct for irregularities in illumination of the microscopic fields. Background levels were equalized and the detection threshold was tested and kept at the same level for all samples.

Statistics. A one-way analysis of variance (ANOVA) was used for somatic measures.

For the acquisition of the MWM, treatment effects were evaluated with a two-factorial (treatment and session) ANOVA with repeated measures over session. For the probe trial, preferences for quadrants were evaluated per group by analyzing with $t$-statistics whether the time spent in a training quadrant, where the platform was located during training, differed from the chance level (15 s).

The data of the testing sessions of the ORT and OF were aggregated per treatment condition to enhance the reliability of the data. A one-factorial ANOVA was used to analyze the effects between groups.

For MAP2 IR and synaptophysin IR, treatment effects were analyzed with a one-way ANOVA $(p<0.05)$.

All statistics were carried out using SPSS software version 12.0.1 (SPSS Inc., Chicago, IL). Data are presented as means \pm SEM.

\section{RESULTS}

Somatic Growth. Betamethasone reduced both body weight, head diameter and crown-tail length at birth $(p<$ 0.001). This difference in size was not observed anymore at 3 wk of age. Female pups were always more affected than males on all parameters $(p<0.001)$. Post hoc analyses per gender are shown in Figure 1.

Morris water maze. Figure $2 A$ depicts the time to find the platform during the acquisition. There was no betamethasone effect except on d $2(p=0.044)$. All experimental groups showed a reduced escape latency over the different days (time effect; $p<0.001)$. In the probe trial, no significant differences were found between groups (Fig. 2B). A training quadrant 

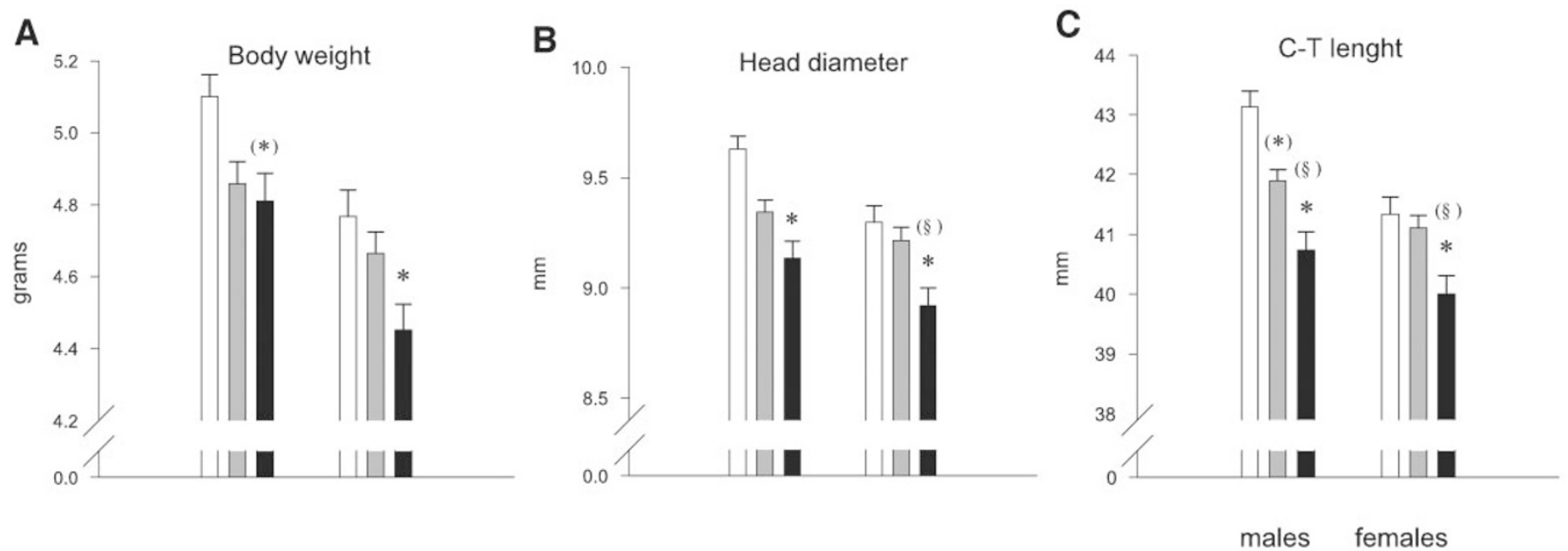

Figure 1. Somatic growth parameters at birth (mean \pm SEM). Vehicle $=$ white bars, $\mathrm{HD}=$ gray bars, $\mathrm{CD}=$ black bars; $n=22-30$. Symbols used for Bonferroni post hoc differences: $(\S) 0.05<p<0.10$ compared with $\mathrm{HD},(*) 0.05<p<0.10$ compared with vehicle group, ${ }^{*} p<0.05$ compared with vehicle group.
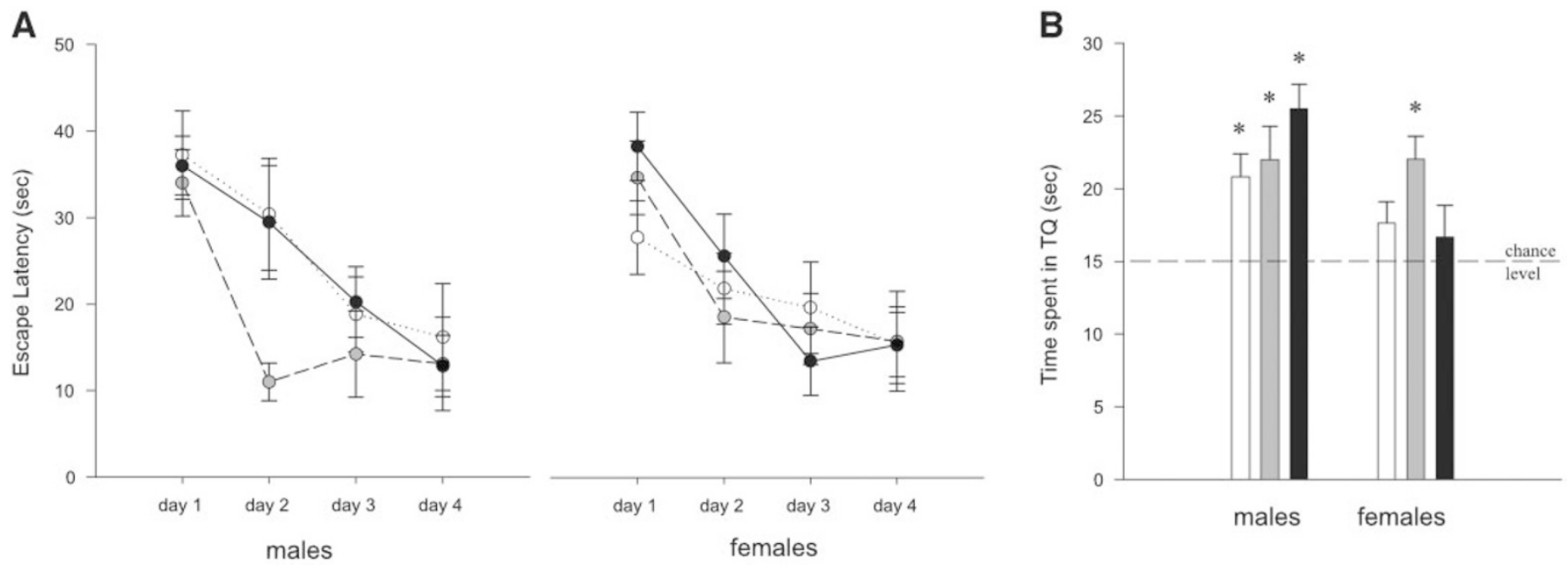

Figure 2. (A) Time spent in the Morris maze during four successive days of training (mean $\pm \mathrm{SEM})$; vehicle $=$ dotted lines, $\mathrm{HD}=$ dashed lines, $\mathrm{CD}=$ solid lines; $n=10$. A treatment effect was found on $\mathrm{d} 2$ combining males and females. $(B)$ Time spent in the training quadrant in the probe trial; $n=10$. A dashed line is drawn at $15 \mathrm{~s}$, which indicates the time that animals would have spent in this training quadrant $(T Q)$ at chance level. ${ }^{*} p<0.05$ compared with chance level.

preference was shown in all the males, whereas in the females only for HD $(p<0.05)$.

Object recognition task. There were no significant differences in E1, E2, and D2 between experimental groups (Fig. 3). However, by comparing each group with the level expected by chance, only CD resulted in higher D2 in the 4-h interval ( $p<$ 0.05 , males and females combined), whereas there was no treatment effect with the 1-h interval.

Open field. The amount of time spent in the different zones of the OF did not differ between groups (data not shown).

Synaptophysin IR and MAP2 IR. Immunohistochemical distribution of synaptophysin IR revealed an extensive and dense granular pattern in the hippocampus, with big clusters of synaptic vesicles in CA3 region. Antenatal betamethasone

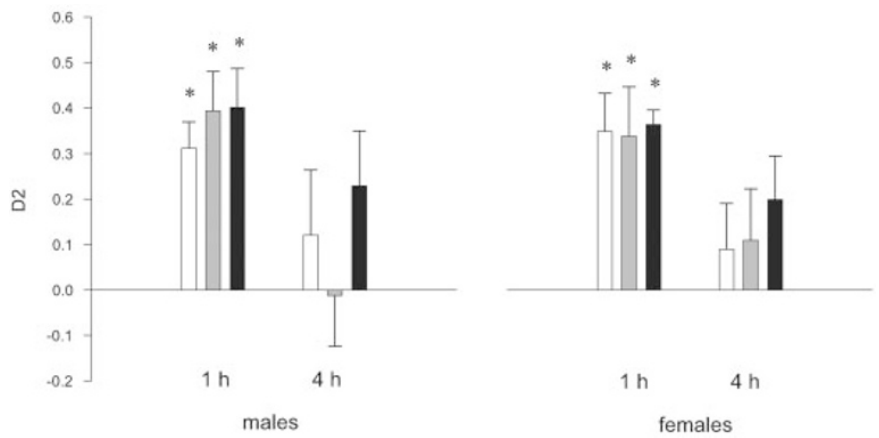

Figure 3. In the ORT, animals were tested at 1 or $4 \mathrm{~h}$ between the first and the second trial. Values are expressed as mean $\pm \mathrm{SEM}$; vehicle $=$ white bars, $\mathrm{HD}=$ gray bars, $\mathrm{CD}=$ black bars $; n=10 . * p<0.05$ compared with chance level, i.e., discrimination index $(\mathrm{D} 2)=0$. 
caused no significant synaptophysin IR changes in the three regions examined (data not shown). Males showed higher values than females in both CA3 and DG $(p<0.001)$.

Exposure to antenatal betamethasone resulted in a tendency toward a significant decrease of MAP2 IR in both the stratum moleculare $(p=0.097)$ and the stratum radiatum $(p=0.099)$ in males (Fig. 4). By comparing treated (CD and $\mathrm{HD}$ ) versus untreated animals, the loss of MAP2 IR by betamethasone was significant in the stratum moleculare $(-18.1 \%, p=0.031)$ and borderline significant in the radiatum $(-15.4 \%, p=0.057)$. Females were not affected by betamethasone treatment. No differences were observed in hippocampus volume.

\section{DISCUSSION}

The present study shows that a single course of antenatal betamethasone impaired fetal growth and MAP2 $\mathrm{R}$ in adult male rats. No significant effect on cognition- and anxietyrelated behavior and synaptophysin IR was observed.

Both body weight, head diameter, and crown-tail length were reduced at birth by a single course of betamethasone equivalent to $12 \mathrm{mg}$ twice $24 \mathrm{~h}$ apart (CD) but not by half of this dose (HD). Corticosteroids have been shown to affect fetal growth, probably reducing the circulating levels of $\mathrm{GH}$ and therefore IGF-I, which are known to stimulate growth (18). In addition, the transplacental transfer of maternal stress hormones, i.e., corticotrophin-releasing factor (CRF) and corticosterone, may affect fetal growth. Moreover, a single course of antenatal betamethasone reduces brain weight in the adult sheep (19) and head diameter in human neonates (2), a finding that has been associated with learning problems in school-age children (20). It is well known that reduced birth size is associated with an increased risk of disease in adulthood (21). Interestingly, this relationship has been suggested to reflect the sensitivity of fetal growth to adverse antenatal events without implying a causal role of being born small, i.e., birth weight may represent a rough integrated measure of intrauterine processes (22). Even the temporary effect on somatic growth reported in this study might therefore have a significant impact on health, as discussed before in more detail $(22,23)$. Of note, in the present study, HD appears to have less influence in terms of growth restriction and the implications mentioned
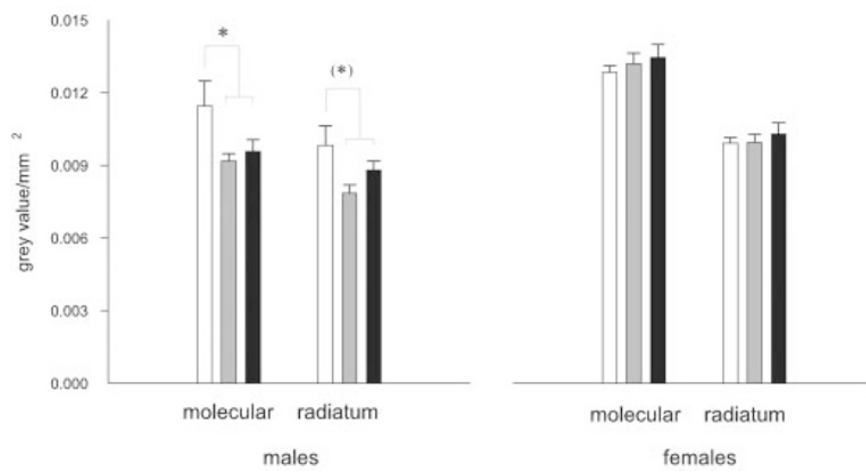

Figure 4. MAP2-IR is expressed as gray value $/ \mu \mathrm{m}^{2}$ (mean $+\mathrm{SEM}$ ); vehicle $=$ white bars, $\mathrm{HD}=$ gray bars, $\mathrm{CD}=$ black bars; $n=10 .{ }^{(*)} 0.05<p<0.10$ betamethasone-treated compared with vehicle; $* p<0.05$ betamethasonetreated compared with vehicle. above when compared with $\mathrm{CD}$, and a clear dose dependency in the effect of antenatal betamethasone on fetal growth was previously described by our group using the same animal model and dose regimen (23).

To our knowledge, this is the first report of long-term effects of antenatal betamethasone on MAP2 IR and synaptophysin IR, shown immunohistochemically in the rat hippocampus at 6 mo of age, which is comparable to an adult human being.

A glucocorticoid effect on MAP2 IR - an acute effect of which has been previously described in the fetal sheep (15) seems to be permanent, suggesting that antenatal betamethasone induces structural alterations in the hippocampus. In rodents, it has been shown that loss of MAP2 IR is associated with neuronal degeneration, which might affect cognition (14). Of note, Di Stefano and colleagues (24) have shown a decrease of MAP2 IR during aging in the rat hippocampus. Thus, loss of MAP2 IR in the hippocampus may be associated with age-related impairment of learning and memory.

Glucocorticoids contribute to brain aging by prolonged exposure to excess glucocorticoid, possibly as a result of decreased glucocorticoid receptors and also altered regulation of neuronal turnover in the DG (25). Cumulative hippocampal changes by corticosteroids lead to abnormal neuroendocrine functioning, cognitive impairment, and increased vulnerability to serious life events in people with mood disorders (26). An increase in synaptophysin IR described with aging suggests a role for synaptophysin in the maintenance of the structural components in the hippocampus (27). The decrease in MAP2 IR was not associated with impairment in learning and memory. However, we cannot exclude that a MAP2 IR decline might result in a cognitive deficit in later life due to aging.

Interestingly, the decrease in MAP2 IR was present in males only. Hippocampal corticosteroid receptor levels are known to be affected in the neonate rat after maternal deprivation (28). Recent evidences suggest that the effects of maternal stress and exogenous glucocorticoids are super imposable (29). Gender-specific stress responses vary depending on developmental age at the time of stress exposure, i.e., male stress responses appear fixed across the lifespan whereas females show a more variable pattern (30). Moreover, recent evidence shows that antenatal glucocorticoids induce genderspecific alterations regarding both cognition and hippocampal cholinergic function in adult rats (31). Steroids affect mineralocorticoid receptor (MR; type I) protein expression within the hippocampus more in males than in females, whereas betamethasone has no effect on glucocorticoid receptor (GR; type II) protein expression (32). This gender differences in the pattern of MR and GR expression during development may result in different periods of vulnerability to glucocorticoid exposure in fetal life. In the present study female rats might be spared at the age of 6 mo by the neuroprotective role exerted by estrogen (33). However, a decline in MAP2 IR might occur when estrogens decrease with aging. This awaits further research.

No differences were observed in synaptophysin IR. Fetal in vivo studies concerning the effect of antenatal betamethasone treatment on synaptophysin showed a reduction in IR after administration of betamethasone (9). However, in those investigations, the animals were analyzed immediately after glu- 
cocorticoid exposure. Taken together, antenatal betamethasone appears to induce an acute synaptophysin IR decrease (9), which does not seem to last, as seen in the present work.

Further, betamethasone treatment did not show any negative effects on adult cognition. Interestingly, discrimination in the ORT was increased by CD, whereas HD somewhat improved spatial learning in the MWM, but only in males. In line with this, antenatal betamethasone has been shown to enhance selective memory in the juvenile mouse, continuing into adulthood in males (34), and improves school behavior in $6 \mathrm{y}$ old girls (35). On the other hand, perinatal steroid administration in the rat has been associated with impaired spatial memory in a dose-dependent manner, though in this study dexamethasone was injected at P4 (36).

In the present study, anxiety-related behavior was not affected by betamethasone in the OF. The same finding was found in the mouse (12), whereas postnatal glucocorticoids resulted in a more anxious behavior in the adult rat (37). This might be due to the permanent changes induced by perinatal glucocorticoid exposure on the anterior pituitary gland (5). In addition, betamethasone effects on anxiety might have been masked by the previous intensive behavioral analysis, i.e., MWM and ORT, with the same strain of rats, as been recently suggested (38).

In summary, we report no detrimental effects of a single course of antenatal betamethasone on cognition- and anxietyrelated behavior and synaptophysin IR in the adult rat. On the other hand, MAP2 IR was decreased in males, suggesting that hippocampus-related cognition may be affected in this gender eventually. Further research might elucidate whether this effect increases with aging and whether both genders are affected in the long run. Finally, HD appears to have less influence in terms of growth restriction when compared with $\mathrm{CD}$, supporting the idea that reducing the dose of antenatal betamethasone might be less harmful $(21,22)$, once clinical trials show its effectiveness for inducing lung maturation.

\section{REFERENCES}

1. [No authors listed] 2000 Antenatal corticosteroids revisited: repeat courses. NIH Consens Statement 17:1-18

2. Thorp JA, Jones PG, Knox E, Clark RH 2002 Does antenatal corticosteroid therapy affect birth weight and head circumference? Obstet Gynecol 99:101-108

3. Kajantie E, Raivio T, Janne OA, Hovi P, Dunkel L, Andersson S 2004 Circulating glucocorticoid bioactivity in the preterm newborn after antenatal betamethasone treatment. J Clin Endocrinol Metab 89:3999-4003

4. Davis EP, Townsend EL, Gunnar MR, Georgieff MK, Guiang SF, Ciffuentes RF, Lussky RC 2004 Effects of prenatal betamethasone exposure on regulation of stress physiology in healthy premature infants. Psychoneuroendocrinology 29:1028-1036

5. Burlet G, Fernette B, Blanchard S, Angel E, Tankosic P, Maccari S, Burlet A 2005 Antenatal glucocorticoids blunt the functioning of the hypothalamic-pituitaryadrenal axis of neonates and disturb some behaviors in juveniles. Neuroscience 133:221-230

6. Pine D, Charney D 2002 Children, stress, and sensitization: an integration of basic and clinical research on emotion? Biol Psychiatry 52:773-

7. Velazquez PN, Romano MC 1987 Corticosterone therapy during gestation: effects on the development of rat cerebellum. Int J Dev Neurosci 5:189-194

8. Scheepens A, van de Waarenburg M, van den Hove D, Blanco CE 2003 A single course of prenatal betamethasone in the rat alters postnatal brain cell proliferation but not apoptosis. J Physiol 552:163-175

9. Colberg C, Antonow-Schlorke I, Muller T, Schubert H, Witte OW, Schwab M 2004 Recovery of glucocorticoid-related loss of synaptic density in the fetal sheep brain at 0.75 of gestation. Neurosci Lett 364:130-134
10. Bruschettini M, van den Hove DL, Gazzolo D, Bruschettini P, Blanco CE, Steinbusch HW 2005 A single course of antenatal betamethasone reduces neurotrophic factor S100B concentration in the hippocampus and serum in the neonatal rat. Brain Res Dev Brain Res 159:113-118

11. Uno H, Lohmiller L, Thieme C, Kemnitz JW, Engle MJ, Roecker EB, Farrell PM 1990 Brain damage induced by prenatal exposure to dexamethasone in fetal rhesus macaques. I.Hippocampus. Brain Res Dev Brain Res 53:157-167

12. Rayburn WF, Christensen HD, Gonzalez CL, Rayburn LA, Stewart JD 1998 Effect of in utero exposure to betamethasone on motivation/anxiety testing in mice offspring. Neurotoxicol Teratol 20:475-481

13. Martinez G, Di Giacomo C, Carnazza ML, Sorrenti V, Castana R, Barcellona ML, Perez-Polo JR, Vanella A 1997 MAP2, synaptophysin immunostaining in rat brain and behavioral modifications after cerebral postischemic reperfusion. Dev Neurosci 19:457-464

14. Matesic DF, Lin RC 1994 Microtubule-associated protein 2 as an early indicator of ischemia-induced neurodegeneration in the gerbil forebrain. J Neurochem 63:1012-1020

15. Schwab M, Antonow-Schlorke I, Kuhn B, Muller T, Schubert H, Walter B, Sliwka U, Nathanielsz PW 2001 Effect of antenatal betamethasone treatment on microtubule-associated proteins MAP1B and MAP2 in fetal sheep. J Physiol 530:497-506

16. Antonow-Schlorke I, Kuhn B, Muller T, Schubert H, Sliwka U, Nathanielsz PW, Schwab M 2001 Antenatal betamethasone treatment reduces synaptophysin immunoreactivity in presynaptic terminals in the fetal sheep brain. Neurosci Lett 297:147-150

17. Clancy B, Darlington RB, Finlay BL 2001 Translating developmental time across mammalian species. Neuroscience 105:7-17

18. Price WA, Stiles AD, Moats-Staats BM, D'Ercole AJ 1992 Gene expression of insulin-like growth factors (IGFs), the type 1 IGF receptor, and IGF-binding proteins in dexamethasone-induced fetal growth retardation. Endocrinology 130:1424-1432

19. Moss TJ, Doherty DA, Nitsos I, Sloboda DM, Harding R, Newnham JP 2005 Effects into adulthood of single or repeated antenatal corticosteroids in sheep. Am J Obstet Gynecol 192:146-152

20. Stathis SL, O'Callaghan M, Harvey J, Rogers Y 1999 Head circumference in ELBW babies is associated with learning difficulties and cognition but not ADHD in the school-aged child. Dev Med Child Neurol 41:375-380

21. Barker DJ 1995 Fetal origins of coronary heart disease. BMJ 311:171-174

22. Gluckman PD, Hanson MA 2004 Developmental origins of disease paradigm: a mechanistic and evolutionary perspective. Pediatr Res 56:311-317

23. Bruschettini M 2006 Lowering the dose of antenatal steroids: the effects of a single course of betamethasone on somatic growth and brain cell proliferation in the rat. Am J Obstet Gynecol 194:1341-1346

24. Di Stefano G, Casoli T, Fattoretti P, Gracciotti N, Solazzi M, Bertoni-Freddari C 2001 Distribution of map2 in hippocampus and cerebellum of young and old rats by quantitative immunohistochemistry. J Histochem Cytochem 49:1065-1066

25. Nichols NR, Zieba M, Bye N 2001 Do glucocorticoids contribute to brain aging? Brain Res Brain Res Rev 37:273-286

26. Brown ES, Rush AJ, McEwen BS 1999 Hippocampal remodeling and damage by corticosteroids: implications for mood disorders. Neuropsychopharmacology 21:474-484

27. Himeda T, Mizuno K, Kato H, Araki T 2005 Effects of age on immunohistochemical changes in the mouse hippocampus. Mech Ageing Dev 126:673-677

28. Sutanto W, Rosenfeld P, de Kloet ER, Levine S 1996 Long-term effects of neonatal maternal deprivation and ACTH on hippocampal mineralocorticoid and glucocorticoid receptors. Brain Res Dev Brain Res 92:156-163

29. Hougaard KS, Andersen MB, Kjaer SL, Hansen AM, Werge T, Lund SP 2005 Prenatal stress may increase vulnerability to life events: comparison with the effects of prenatal dexamethasone. Brain Res Dev Brain Res 159:55-63

30. Bowman RE 2005 Stress-induced changes in spatial memory are sexually differentiated and vary across the lifespan. J Neuroendocrinol 17:526-535

31. Kreider ML, Levin ED, Seidler FJ, Slotkin TA 2005 Gestational dexamethasone treatment elicits sex-dependent alterations in locomotor activity, reward-based memory and hippocampal cholinergic function in adolescent and adult rats. Neuropsychopharmacology 30:1617-1623

32. Owen D, Matthews SG 2003 Glucocorticoids and sex-dependent development of brain glucocorticoid and mineralocorticoid receptors. Endocrinology 144:2775-2784

33. Norbury R, Cutter WJ, Compton J, Robertson DM, Craig M, Whitehead M, Murphy DG 2003 The neuroprotective effects of estrogen on the aging brain. Exp Gerontol 38:109117

34. Rayburn WF, Christensen HD, Gonzalez CL 1997 A placebo-controlled comparison between betamethasone and dexamethasone for fetal maturation: differences in neurobehavioral development of mice offspring. Am J Obstet Gynecol 176:842-850

35. MacArthur BA, Howie RN, Dezoete JA, Elkins J 1982 School progress and cognitive development of 6-year-old children whose mothers were treated antenatally with betamethasone. Pediatrics 70:99-105

36. Vicedomini JP, Nonneman AJ, DeKosky ST, Scheff SW 1986 Perinatal glucocorticoids disrupt learning: a sexually dimorphic response. Physiol Behav 36:145-149

37. Neal CR Jr, Weidemann G, Kabbaj M, Vazquez DM 2004 Effect of neonatal dexamethasone exposure on growth and neurological development in the adult rat. Am J Physiol Regul Integr Comp Physiol 287:R375-R385

38. Van den Hove DL, Blanco CE, Aendekerk B, Desbonnet L, Bruschettini M, Steinbusch HP, Prickaerts J, Steinbusch HW 2005 Prenatal restraint stress and long-term affective consequences. Dev Neurosci 27:313-320 